\title{
Mean Scatterer Spacing Estimation Using Wavelet Spectrum
}

\author{
Jenho Tsao and Guang-Shiuan Jiang \\ Dept. of EE. And Inst. of Comm., National Taiwan University, Taipei, Taiwan, ROC.
}

\begin{abstract}
Mean scatterer spacing (MSS) has been proven as an effective method for characterizing biological tissue structures, especially for liver tissue. Periodogram, correlogram, cepstrum and spectral autocorrelation (SAC) are the major techniques proposed for MSS estimation. However, due to the interferences of diffusive scattering echo, it is difficult to estimate the MSS of the regular (quasi-periodic) scatterers. Robust techniques, such as the SAC method, which can tolerate the diffusive speckle interference are preferred. A newly developed periodicity detection technique known as Periodic Wavelet Transform (PWT) is proposed for MSS estimation here. Wavelet spectrum is defined as the PWT over scale space. The wavelet approach provides an unique framework for the MSS estimation problem.
\end{abstract}

\section{INTRODUCTION}

Spectral analysis of tissue echo is a major approach to ultrasonic tissue characterization [1]. The backscattered signal of soft tissues can be modeled as a composition of coherent (regular) and diffusive components (speckle) [1-5]. Inter-spacing of the coherent scatterers is essentially random[4], their mean value is known as mean scatterer spacing (MSS). Depending on the regularity of tissue structure, echo of the coherent scatterers has different degree of periodicity and is known as quasi-periodic signal. MSS has been proven to be an effective method for characterizing the underlying scattering structures of soft tissue. Periodogram, correlogram, cepstrum and spectral autocorrelation (SAC) [1-3] are some of the techniques proposed for MSS estimation. However, due to interference of the diffusive scattering components and the quasi-periodic nature of the coherent scatterers, it is difficult to estimate the MSS of the coherent scatterers reliably. Robust techniques, such as the SAC method, which can tolerate the diffusive speckle interference are preferred. A newly developed periodicity detection technique known as Periodic Wavelet Transform (PWT) is proposed for MSS estimation.

This work is supported by the National Science Council, Taiwan, ROC. (NSC93-2213-E002-082)
PWT is a generalization of Fourier transform (FT), their differences are : the transformation kernel of PWT is a concatenation of wavelet basis instead of sinusoidal basis in FT, and the spectrum is calculated in scale space instead of in frequency space. PWT is introduced for periodicity detection by Benedetto et. al.[6]. PWT can be designed to use optimal wavelet basses for detecting the periodicity of signals, which must be constructed from the signal to be transformed. Since the basis is constructed under the hypothesis that the signal is periodic and contaminated by noise, it will neglect the non-periodic components as much as possible. Thus PWT is an adaptive and robust generalized spectral analysis technique.

The PWT introduced here is designed mainly for the MSS estimation problem. For clarity, some notations of wavelets are simplified. Theoretical works and experimental demonstrations are given.

\section{THEORIES}

\section{A. Model of backscattered signal}

Empirically the scattering function of soft tissue can be modeled as

$$
\begin{aligned}
\eta(t) & =\eta_{C}(t)+\eta_{D}(t) \\
& =\sum_{i} u_{i}\left(t-\xi_{i}\right)+\sum_{j} v_{j}\left(t-\zeta_{j}\right)
\end{aligned}
$$

where $u_{i}(t)$ is the scattering function of the $i^{t h}$ coherent scatterer with delay $\xi_{i}$ and $v_{j}(t)$ is the scattering function of the $j^{\text {th }}$ diffusive scatterer with delay $\zeta_{j}$. The temporal spacing between two consecutive coherent scatterers can be defined as a random variable $\tau$, with a pdf $f_{\tau}(\tau)$, which has sample values of $\left(\xi_{i}-\xi_{i-1}\right)$ [4]. Then the temporal mean scatterer-spacing can be defined as $\mathrm{MSS}_{\mathrm{T}}=\bar{\tau}$ and the spatial mean scatterer-spacing can be defined as $\mathrm{MSS}=\mathrm{C} \bar{\tau} / 2$, where $C$ is the propagation velocity of the medium. Let the two-way impulse response of the interrogation system be $h(t)$, which is essentially range dependent. However, 
usually the signal segment to be processed is short, the system impulse response could be assumed to be shift invariant within the segment. Thus, the backscattered signal can be modeled as $x(t)=\eta(t) * h(t)$. When scatterer size is small relative the system range resolution, the scatterers can be approximated to be point scatterers with different scattering amplitudes and time invariant scattering function $\delta(t)$. Then the coherent backscattered signal can be written to be

$$
\begin{aligned}
x_{C}(t) & =h(t) * \eta_{C}(t) \\
& =h(t) * \sum_{i} a_{i} \delta\left(t-\xi_{i}\right)=\sum_{i} a_{i} h\left(t-\xi_{i}\right)
\end{aligned}
$$

where $a_{i}$ is the scattering amplitude of the $i^{t h}$ coherent scatterer. The diffusive backscattered component can be written to be

$$
\begin{aligned}
n_{D}(t) & =h(t) * \eta_{D}(t) \\
& =h(t) * \sum_{j} b_{j} \delta\left(t-\zeta_{j}\right)=\sum_{j} b_{j} h\left(t-\zeta_{j}\right),
\end{aligned}
$$

where $b_{j}$ is the scattering amplitude of the $j^{\text {th }}$ diffusive scatterer. Then we have the RF backscattered signal $x(t)=x_{C}(t)+n_{D}(t)$. For MSS estimation problem, the diffusive component $n_{D}(t)$ is considered to be speckle interferences.

\section{B. The Periodic Wavelet Transform}

\section{Coherent PWT}

PWT is a generalization of Fourier transform. Each period of Fourier basis is a sinusoid. Similarly, each period of PWT basis is a wavelet $\psi(t)$, which is known as the mother wavelet in the literature of wavelet transform. The scaled and delayed versions of mother wavelet, $\psi_{n}(t)=\psi\left(t-t_{n}\right)$, are used as the wavelet basis set for wavelet transform. A major purpose of using wavelet transform is to extract the local properties of a signal through inner product to the wavelet basis. For analyzing finite-length signals, the PWT is defined to have finite length here.

An order-Q periodic wavelet basis is defined as

$$
\begin{aligned}
\phi_{Q}(t) & =\sum_{q=1}^{Q} \psi_{q}(t)=\sum_{q=1}^{Q} \psi\left(t-t_{q}\right) \\
& =\sum_{q=1}^{Q} \psi\left(t-q T-t_{0}\right)
\end{aligned}
$$

It consists of $Q$ sub-wavelets $\psi_{q}(t)$ located at $t_{q}=q T+t_{0}, q=1,2, \ldots, Q$. In the terminology of wavelet transform, $T$ is a scaling (dilation) parameter and $t_{q}$ is a translation (delay) parameter of the periodic wavelet basis. An example of periodic wavelet basis with order $Q=5$ is shown in Fig.1(b), which is constructed from the usual wavelet basses given in Fig.1(a). To detect the coherent scatters optimally, the sub-wavelet is defined to be the system impulse response here. That is, $\psi_{q}(t)=h\left(t-t_{q}\right)$, which is assumed to be known and be a normalized complex basis.

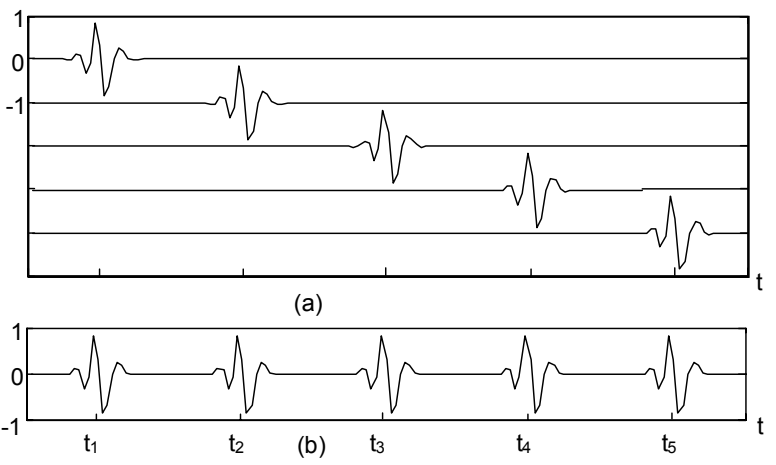

Fig.1 (a) Five example wavelet basses. (b) An order-5 periodic wavelet basis constructed from the basses in (a).

The wavelet spectrum is defined by inner product as

$$
X_{W}(T)=\left\langle x_{G}(t), \phi_{Q}(t)\right\rangle=\frac{1}{Q} \sum_{q=1}^{Q}\left\langle x_{G}(t), \psi_{q}(t)\right\rangle
$$

where $x_{G}(t)$ is the gated signal segment for doing periodic wavelet transform. $x_{G}(t)$ is assumed to be in its analytic form with complex values. To do PWT, the parameter $t_{0}$ must be given and $x_{G}(t)$ must have a length larger enough to accommodate at least $Q+1$ sub-wavelets for the following reason.

To use PWT as a means for MSS estimation, it is required that a coherent scatterer is located at $t=t_{0}$. One way to find $t_{0}$ is to search it by a coherent scatterer detector, which ensures that each sub-wavelet is synchronized to a coherent scatterer in the signal segment. The coherent scatterer located at $t=\xi_{0}$ is referred to as a reference scatterer and the sub-wavelet located at $t=t_{0}=\xi_{0}$ is referred to as a reference wavelet. Note the reference wavelet is not shown in Fig.1. 


\section{Incoherent PWT}

PWT might be thought as a matching procedure for periodic wavelets with different scale $T$. It is sensitive to the phase of each local matched-filter defined by the sub-wavelets. Since the phase of coherent scatterers are not interested in MSS estimation, an incoherent PWT is defined for MSS estimation. The incoherent wavelet spectrum $X_{w w}(T)$ is defined via its energy spectrum as

$$
X_{w w}^{2}(T)=\frac{1}{Q} \sum_{q=1}^{Q}\left\|\left\langle x_{G}(t), \psi_{q}(t)\right\rangle\right\|^{2} .
$$

\section{PWT for MSS Estimation}

\section{PWT of periodic signal}

When the coherent scatterers are located periodically at $\xi_{i}=i T_{c}+\xi_{0}, x_{C}(t)$ is a periodic wavelets. It can be expected that the wavelet spectrum $X_{W}(T)$ will have a strong peak at $T=T_{c}$, where $T_{c}$ is the inter-spacing of coherent scatterers. Thus PWT can serve as a means for periodicity detection and period estimation. To achieve the maximum SIR gain of $X_{W}(T)$ at $T=T_{c}$, it is required that $t_{0}=\xi_{0}$, which ensures that all sub-wavelets in $\phi_{Q}(t)$ can be matched to coherent scatterers to achieve the desired SIR gain of the Q matched filters in PWT. Compared to FFT, a major advantage of PWT is that the periodic wavelet basis is designed to be the matched filter of $x_{C}(t)$, it maximize the SIR of wavelet spectrum at $T=T_{c}$. A simulation example is given in Fig. 2 and Fig. 3 for $Q=5$ and $S I R=3$ and $-3 d B$.



Fig. 2 A set of simulation signals. Top: $x_{c}(t)$, bottom: $x_{G}(t)$ with $S I R=-3 d B$ and $T c=30 T s$.

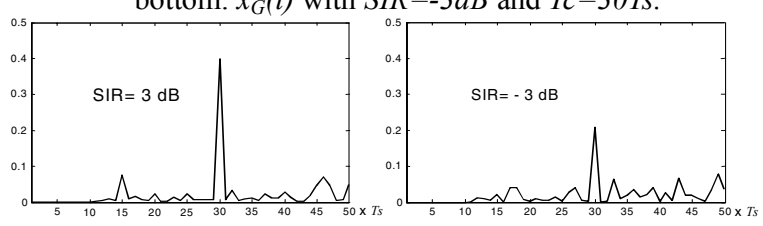

Fig. 3 The coherent wavelet spectrum for $Q=5$

\section{PWT of quasi-periodic signal}

For backscattered signal of soft tissues, the locations of coherent scatterers are not exactly periodic. $x_{C}(t)$ can only be a quasi-periodic signal. In this case, the wavelet spectrum will be deteriorated depending on the regularity of tissue structure. A critical point in using PWT for scatterer-spacing estimation is to know what wavelet spectrum means when signal is not exactly-periodic as hypothesized in the design of PWT.

Since PWT is simply a matched filter for "a pattern of Q sub-wavelets", wavelet spectrum can be thought as a pattern spectrum. Based on the concept of pattern spectrum, The ensemble average of squared wavelet spectrum can be defined as wavelet power spectral density (PSD) as : $P_{W}(T)=E\left[\left|X_{W}(T)\right|^{2}\right]$ for the coherent PWT, or $P_{W}(T)=E\left[X_{w w}^{2}(T)\right]$ for the incoherent PWT. Thus the normalized wavelet power spectral density represents the percentage of total signal power that contributes to the periodic wavelet basis (patterns) $\phi_{Q}(t)$ with period $T$. It can be shown that the order-1 normalized wavelet PSD is an estimate of $f_{\tau}(\tau)$. Therefore $P_{W}(T)$ can be treated as the likelihood of " $M S S_{T}=T$ ", then the MLE of $M S S_{T}$ is $T_{M L E}=\arg \max \left\{P_{W}(T)\right\}$ and a possible decision variable for periodicity detection is

$$
\Lambda(T)=\frac{\text { peak of } P_{W}(T)}{\text { averageof } P_{W}(T) \text { with peaksuppressed }} .
$$

If $f_{\tau}(\tau)$ is unimodal and symmetrical, $T_{M L E}$ would be equivalent to the expected value of $\tau$, i.e., $T_{M L E}=E[\tau]$.

\section{EXPERIMENTAL STUDIES}

In Vitro RF pulse echo of sliced pig liver interrogated in water tank by a broadband $5 \mathrm{MHz}$ transducer is sampled by a 8 -bit, $20 \mathrm{MHz}$ A/D converter for MSS estimation. A segment of echo with a length of 350 samples $(=17.5 \mu \mathrm{s}$ with $T s=0.05 \mu s$ or $13.3 \mathrm{~mm}$ ) is used for finding the wavelet spectral density. This test signal is shown in Fig.4. This is a case with coherent scatterers being well resolved and suitable for PWT. The two-way impulse response measured as the echo of 
a flat reflector is used in designing the PWT. The impulse response has about $70 \%$ bandwidth, which is used as the example wavelet in Fig.1.

The normalized wavelet power spectral density functions, using incoherent PWT with order $Q=1$ and 10, are shown in Fig.5. The period of PWT is sampled, with the same spacing as the RF data, from $T=11 T s$ to $T=50 T s$.

For $Q=1$, the wavelet spectral density function is an estimate of scatterer-spacing density $f_{\tau}(\tau)$,which is shown in Fig.5(a). Its has a mean value of $32.1 T s$, which corresponds to MSS $=1.2 \mathrm{~mm}$, and the standard deviation of scatterer-spacing is $\sigma_{S S}=8 T s$. A tissue regularity index can be defined as $\sigma_{S S} / M S S=25 \%$, which is considered to be large and makes MSS estimation difficult. An additional result shown in Fig.5 is that $f_{\tau}(\tau)$ may not be unimodal and symmetrical.

For $Q=10$, this is the largest order allowable for the test signal. The wavelet spectrum is shown in Fig.5(b), like an FFT using longer basis, its spectral resolution is higher than the $Q=1$ case, and has spurious sidelobes. Since this wavelet PSD is estimated based on a single realization of sample signal, its sidelobe is quite high. However, the spectral peak is still apparent and $T_{M L E}=31 T \mathrm{~s}$, which corresponds to $M S S=1.18 \mathrm{~mm}$. Two sources for the sidelobes are mismatched coherent scattering signals and speckle interferences.

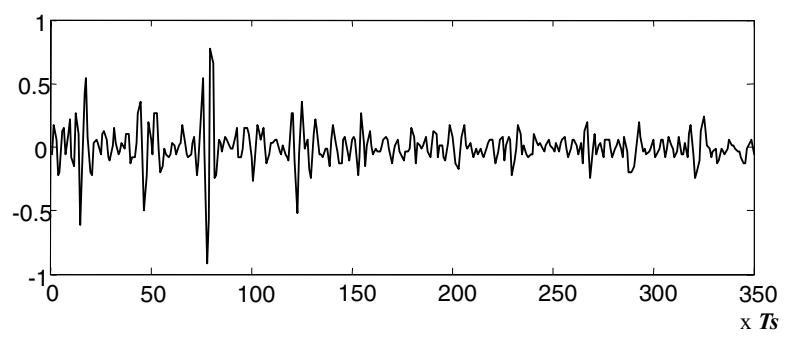

Fig.4 The backscattering signal of pig liver sampled with $T s=0.05 \mu s$.

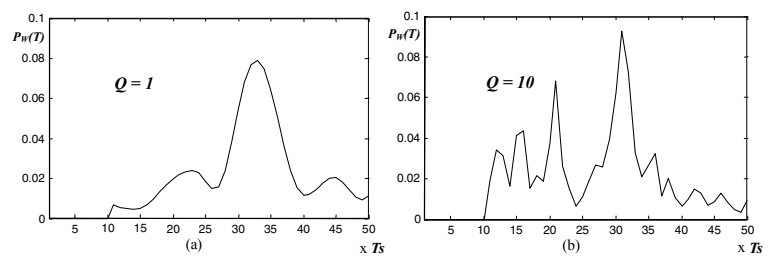

Fig.5 The normalized wavelet power spectral density function, using incoherent PWT with order $Q=1$ and 10 .

\section{DISCUSSION AND CONCLUSION}

In this paper, only some introductory materials are given, which is far from being complete. Detail proofs of the theoretical results and computer simulations for accessing the performance of PWT will be given elsewhere.

The quality of the experimental signal selected for this study is considered to be very good, since most of the backscattering signals collected do not have such apparent responses of coherent scatterers. To allow MSS estimation, the periodicity must be tested first, therefore periodicity detection is more important than MSS estimation. Some additional conclusions are given below.

PWT provides a scheme for simultaneous periodicity detection and scatterer-spacing density estimation, which is more than a parameter of MSS.

Wavelet spectrums of different orders may provide another measures of tissue regularity.

Compared to FT, PWT is more flexible, in which the coherent scatterers can be processed independently by sub-wavelets. The incoherent PWT is an example for this flexibility.

\section{References :}

[1] L. L. Fellingham, F. G. Sommer, "Ultrasonic characterization of tissue structure in the in vivo human liver and spleen", IEEE Trans. Sonics Ultrson., vol.SU-31, no.4, pp.418-428, 1984.

[2] K. Wear, R. Wagner, M. Insana, T. Hall, "Application of autoregressive spectral analysis to cepstral estimation of mean scatterer spacing", IEEE Trans. Ultrason., Ferroelect., Freq. Contr., vol.40, pp.50-58, Jan. 1993.

[3] T. Varghese, K. Donohue, "Estimating mean scatterer spacing with the frequency-smoothed spectral autocorrelation function", IEEE Trans. Ultrason., Ferroelect., Freq. Contr., vol.42, pp.451-463, May 1995.

[4] L. Landini, L. Verrazzani, "Spectral characterization of tissue microstructure by ultrasound: A stochastic approach", IEEE Trans. Ultrason., Ferroelect., Freq. Contr., vol.37, pp.448-456, 1990.

[5] F.S. Cohen, G. Georgiou, "Bayesian detection and estimation of regularity in soft tissue organs", 1996 IEEE Ultrasonics Symposium, Nov. 1996, Proceedings., Vol.2, pp.1089-1092.

[6] J.Benedetto and G. Pfander,"Periodic wavelet transforms and periodicity detection", Society for Industrial and Applied Mathematics, pp. 1329-1367, 2002.

E-mail: tsaor215@cc.ee.ntu.edu.tw 